\title{
Quantitative precision of optical frequency domain imaging: direct comparison with frequency domain optical coherence tomography and intravascular ultrasound
}

\author{
Yuhei Kobayashi $^{1} \cdot$ Hideki Kitahara $^{1} \cdot$ Shigemitsu Tanaka $^{1} \cdot$ Kozo Okada $^{1}$. \\ Takumi Kimura $^{1} \cdot$ Fumiaki Ikeno $^{1} \cdot$ Paul G. Yock $^{1} \cdot$ Peter J. Fitzgerald $^{1}$ • \\ Yasuhiro Honda ${ }^{1}$
}

Received: 12 June 2015/Accepted: 1 August 2015/Published online: 14 August 2015

(C) Japanese Association of Cardiovascular Intervention and Therapeutics 2015

\begin{abstract}
No systematic validation study is available with optical frequency domain imaging (OFDI), directly compared with frequency domain optical coherence tomography (FD-OCT) and intravascular ultrasound (IVUS). Controversy also remains about the impact of different stent contour tracing methods by OFDI/FD-OCT. In vitro: coronary phantom models $(1.51-5.04 \mathrm{~mm})$ were imaged with OFDI, FD-OCT, and IVUS, demonstrating excellent quantitative precision with a slight overestimation of mean lumen diameter (difference 0.01-0.02 mm). In vivo: corresponding 64 OFDI/IVUS images of stented coronary segments from 20 swines were analyzed. Minimum lumen area by OFDI was larger than IVUS at baseline $(P<0.001)$, whereas it was smaller than IVUS at followup. When stent was traced at leading edges of struts by OFDI, minimum stent area was similar between OFDI and IVUS $(P=0.60)$. When traced at the highest intensity points of struts by OFDI, it was significantly larger in OFDI than in IVUS $(P<0.001)$. Three modalities have clinically acceptable precision across the wide range of lumen diameters. In vivo measurements by OFDI and IVUS could slightly be discrepant depending on the parameters and time points. In stent assessment by OFDI, the 2 methods led to a small but systematic difference; therefore, consistency in methodology is advised for comparative studies.
\end{abstract}

Yasuhiro Honda

crci-cvmed@stanford.edu

1 Division of Cardiovascular Medicine, Stanford Cardiovascular Institute, Stanford University School of Medicine, 300 Pasteur Drive, Room H3554, Stanford, CA 94305-5637, USA
Keywords Intravascular ultrasound · Optical frequency domain imaging $\cdot$ Imaging

$\begin{array}{ll}\text { Abbreviations } \\ \text { FD-OCT } & \begin{array}{l}\text { Frequency domain optical coherence } \\ \text { tomography }\end{array} \\ \text { IVUS } & \begin{array}{l}\text { Intravascular ultrasound } \\ \text { LD }\end{array} \\ \text { MHz } & \text { Megahen diameter } \\ \text { OCT } & \text { Optical coherence tomography } \\ \text { OFDI } & \text { Optical frequency domain imaging } \\ \text { VI } & \text { Volume index }\end{array}$

\section{Introduction}

Time domain and frequency domain (FD) optical coherence tomographies (OCTs) have been consistently shown to provide more precise lumen measurements compared to conventional 40-megahertz (MHz) intravascular ultrasound (IVUS) both in vitro and in vivo, owing to their much higher resolution and better contrast of lumen border than IVUS systems [1-3].

Recently, optical frequency domain imaging (OFDI) has become clinically available as another second-generation intravascular optical imaging system. While a first-in-human study demonstrated the feasibility and safety of OFDI, [4] there had been no systematic validation study directly comparing OFDI with FD-OCT. Although these secondgeneration OCT systems adopt the same technical principle, there is a theoretical concern that practical differences in image data processing and system configurations may lead to considerable variability in quantitative precision, as previously demonstrated in IVUS systems [5, 6]. Also, it 
remains controversial whether stent contour should be traced at leading edges or the highest intensity points when assessing with intravascular optical imaging systems [7]. Accordingly, the primary goal of the present study was to compare the precision of OFDI with FD-OCT and IVUS. Additionally, the secondary goal was to compare OFDI stent measurements between tracing at leading edges and the highest intensity points.

\section{Methods}

This study consisted of an in vitro phantom study that compared OFDI with FD-OCT and IVUS, and an in vivo swine study that compared OFDI and IVUS. All OFDI, FD-OCT, and IVUS images obtained in a DICOM format were analyzed offline by a dedicated interpreter (Y. K.) and reviewed by another independent analyst (H. K.) at Stanford Cardiovascular Core Analysis Laboratory using a commercially available image analysis system (echoPlaque $^{\text {TM}}$; INDEC Medical Systems, Santa Clara, CA). IVUS data were blinded to interpreters at the time of OFDI analysis.

\section{In vitro study}

Five coronary phantom models made from an acrylic resin with known lumen diameters $(1.51,2.03,3.04,4.04$, and $5.04 \mathrm{~mm}$, respectively) were imaged with OFDI (FastView $^{\mathrm{TM}}$ and LUNAWAVE ${ }^{\mathrm{TM}}$, Terumo Corp., Tokyo, Japan), FD-OCT (Dragonfly ${ }^{\mathrm{TM}}$ JP and ILUMIEN OPTIS $^{\text {TM }}$, St. Jude Medical, MN), and IVUS (ViewIT ${ }^{\circledR}$ and VISIWAVE ${ }^{\mathrm{TM}}$, Terumo Corp.) catheter systems. OFDI and FD-OCT images were obtained using automated motorized pullback in a tank filled with 37 degrees Celsius saline, saline $50 \% /$ contrast $50 \%$, and contrast $100 \%$ under the system setting of the refractive index for the corresponding flush medium. To avoid possible variability caused by varying blood characteristics, IVUS imaging was performed under standardized conditions in the tank filled with 37 degrees Celsius saline using automated motorized pullback [6]. In each phantom diameter of each modality, 10 analyzable cross sections were randomly selected, and phantom lumen contours were traced. Mean lumen diameter (LD) of the analyzed cross section was provided automatically by the analysis system. Because IVUS measurements assume imaging in a blood environment, obtained data were adjusted according to the speed of sound in the 37 degrees Celsius saline $(1522.3 \mathrm{~m} / \mathrm{s})$ [8] and the theoretical speed of sound in blood employed in the IVUS system $(1562.5 \mathrm{~m} / \mathrm{s})$, as well as the catheter diameter at the imaging window level $(0.87 \mathrm{~mm})$. The catheter diameter on a cross-sectional image is predetermined by the system irrespective of a medium used. Accordingly, the following formula was used to compensate the difference of speed of sound between saline and blood (Fig. 1): adjusted diameter $=$ (measured diameter - catheter diameter $) \times($ speed of sound in saline/speed of sound in blood + catheter diameter.

\section{In vivo study}

In vivo OFDI/IVUS images of stented coronary segments were obtained from 20 swines. Dual antiplatelet therapy with aspirin $325 \mathrm{mg} /$ day and clopidogrel $75 \mathrm{mg} /$ day was initiated 3 days before the stent deployment and continued indefinitely. After injection of $200 \mu \mathrm{g}$ intracoronary nitroglycerin, left and right coronary angiograms were performed to assess diameter and length of a given coronary segment. If stent deployment was feasible, the type of stent was randomly selected from the following 5 stents: Ultimaster $^{\mathrm{TM}}$, Nobori ${ }^{\mathrm{TM}}$, and Kaname ${ }^{\mathrm{TM}}$ (Terumo Corp.); Xience Prime ${ }^{\mathrm{TM}}$ (Abbott Vascular, Inc., Santa Clara, CA); and Resolute Integrity ${ }^{\mathrm{TM}}$ (Medtronic Vascular, Inc., Santa Rosa, CA). To achieve a stent/arterial ratio of 1.1-1.2, stent diameter was selected as either 2.50 or $3.00 \mathrm{~mm}$, and stent deployment pressure was determined according to the manufacturers' compliance charts.

OFDI imaging of the stented segment was performed under flushing of an optically transparent media at $4 \mathrm{ml} / \mathrm{s}$ for $4 \mathrm{~s}$ (contrast, crystalloid, or the mixture of contrast and crystalloid: randomly selected under the system setting of the refractive index for the corresponding flush medium.) using the FastView ${ }^{\mathrm{TM}}$ catheter with automated motorized pullback at $20 \mathrm{~mm} / \mathrm{s}$. IVUS imaging of the same segment was obtained in a standard fashion using the ViewIT ${ }^{\circledR}$

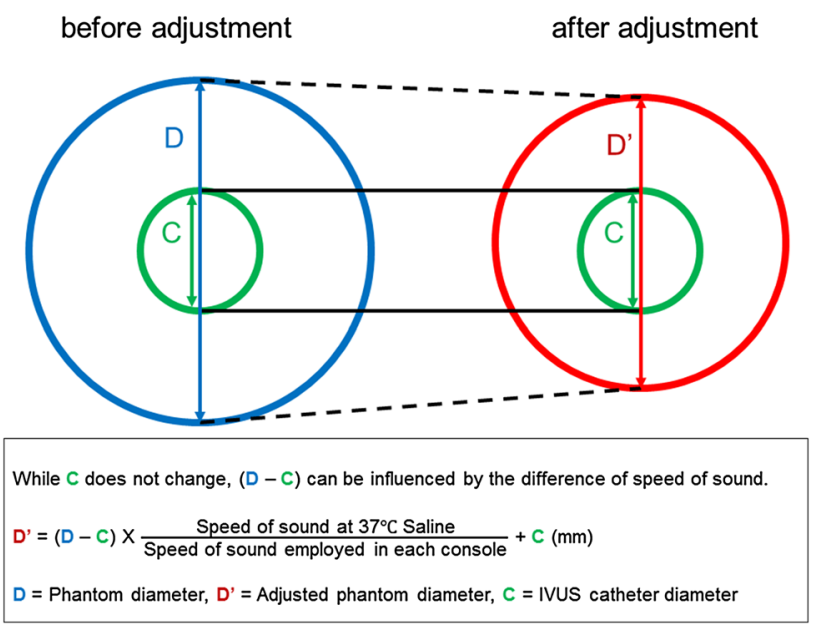

Fig. 1 The scheme of IVUS data adjustment. Because IVUS systems are optimized for measurements in the blood, obtained IVUS data were adjusted to compensate for the difference in the speed of sound in saline and blood. IVUS intravascular ultrasound 
catheter with automated motorized pullback at $0.5 \mathrm{~mm} / \mathrm{s}$. Stents were imaged again with OFDI and IVUS at either 4 or 12 weeks after the stent deployment [9].

Lumen and stent contours were traced at 1-mm axial intervals for the stented segment to calculate the volumes using Simpson's rule with automated frame interpolation by the analysis system. All IVUS measurements were performed at the leading edge of boundaries, according to the American College of Cardiology Clinical Expert Consensus Document on Standards for Acquisition, Measurement, and Reporting of Intravascular Ultrasound Studies [10]. All OFDI analyses were performed according to the Consensus Standards for Acquisition, Measurement, and Reporting of Intravascular Optical Coherence Tomography Studies [7]. Of note, in assessing lumen contours at baseline, the lumen border behind the stent struts was traced in OFDI images, whereas by IVUS, lumen contour was equal to stent contour in absence of incomplete strut apposition (malapposition). In-stent measurements by OFDI, both leading edges and the highest intensity points of stent strut images were traced to determine the exact degree of difference between the 2 methods (Fig. 2) [7, 10]. At followup, neointimal volume was calculated as stent volume minus lumen volume. Each volume was divided by an axial analyzed length (volume index: VI, $\mathrm{mm}^{3} / \mathrm{mm}$ ) to adjust for the difference of analyzed longitudinal lengths among cases. Minimum lumen area and mean LD of the minimum lumen area site were also obtained as 2-dimensional indices.

\section{Intra- and interobserver reproducibility}

Reproducibility of the in vitro study was assessed in 50 cross-sections in each modality at least 4 weeks later after the initial evaluation with the phantom. Intra- and interobserver variability of the in vivo study was assessed in randomly selected 6 OFDI and 6 IVUS pullbacks by the dedicated interpreter (Y.K.) and an independent 2nd interpreter (S.T.).

\section{Statistical analysis}

All analyses were performed using SPSS 21 software $^{\circledR}$ (IBM Corp., Armonk, NY). Continuous variables are presented as mean \pm standard deviation. Normality of the continuous variables was confirmed with Shapiro-Wilk test. Two sets of data with normal distribution were compared with paired Student $t$ test. If the normality test failed, they were compared using the Wilcoxon signed-rank test. Bland-Altman method [11] was employed to test the agreement between the 2 modalities. The degree of reproducibility was presented with a mean difference \pm standard deviation and a coefficient of variation (defined as the standard deviation of the paired difference divided by the mean of the absolute value) [12,13]. A twosided $P$ value of $<0.05$ was considered significant.

\section{Results}

\section{In vitro study}

A total of 150 cross sections were analyzed for the in vitro study. Mean LD obtained in saline for the 3 modalities as well as adjusted values for IVUS are summarized in Table 1. Saline-blood adjustments for IVUS data led to smaller mean LD; however, \%change of mean LD after adjustments was diminutive, ranging from -2.14 to $-1.27 \%$. Mean LDs by OFDI and FD-OCT in saline, saline $50 \% /$ contrast $50 \%$, and contrast $100 \%$ are summarized in Table 2. The difference of flushing media had only minimal impact on measurements (ranging from 0.20 to $3.36 \%$ change of mean $\mathrm{LD}$ ), as long as the reflective index of flushing media was adjusted by the system setting. Overall, all modalities demonstrated excellent quantitative precision with only a slight overestimation of mean LD (difference of each modality: $0.02 \pm 0.02 \mathrm{~mm}$ for OFDI, $0.01 \pm 0.02 \mathrm{~mm}$ for FD-OCT, and $0.02 \pm 0.02 \mathrm{~mm}$ for IVUS, respectively) (Fig. 3).

Reproducibility of the in vitro study was high, irrespective of modality. Mean difference between 1st and 2nd analysis and coefficient of variation in each modality was $0.00 \pm 0.01 \mathrm{~mm}$ and $0.27 \%$ for OFDI, $0.01 \pm 0.02 \mathrm{~mm}$ and $0.55 \%$ for FD-OCT, and $0.02 \pm 0.02 \mathrm{~mm}$ and $0.52 \%$ for IVUS, respectively.

\section{In vivo study}

OFDI and IVUS images were both obtained in a total of 38 stented coronary segments, 26 of which had serial studies performed at baseline and follow-up. In total, 34 corresponding OFDI/IVUS images were available at baseline immediately after stent deployment, 17 images at 4 weeks, and 13 images at 12 weeks.

Detailed in vivo measurement results are summarized in Table 3 and Fig. 4. For lumen assessment, OFDI measurements were significantly larger than IVUS at baseline $(P<0.001$ for all), whereas those at 4 weeks and 12 weeks were similar or significantly smaller than IVUS. For stent assessment, when traced at leading edges of strut images by OFDI, overall stent measurements were similar between OFDI and IVUS (difference in minimum stent area $=-0.02 \pm 0.28 \mathrm{~mm}^{2}, P=0.60$; difference in stent $\mathrm{VI}=-0.03 \pm 0.26 \mathrm{~mm}^{3} / \mathrm{mm}, P=0.39$ ). When the stent was traced at highest intensity points of strut images by OFDI, OFDI stent measurements were significantly larger 
Fig. 2 Representative images of lumen and stent trace in the same segment. Yellow and green lines represent lumen and stent contour. In the baseline IVUS image, lumen and stent contour were traced at the same line. IVUS intravascular ultrasound, $O F D I$ optical frequency domain imaging. HIP and $L E$ indicate stent tracings in OFDI image were performed either at the highest intensity points or at leading edges
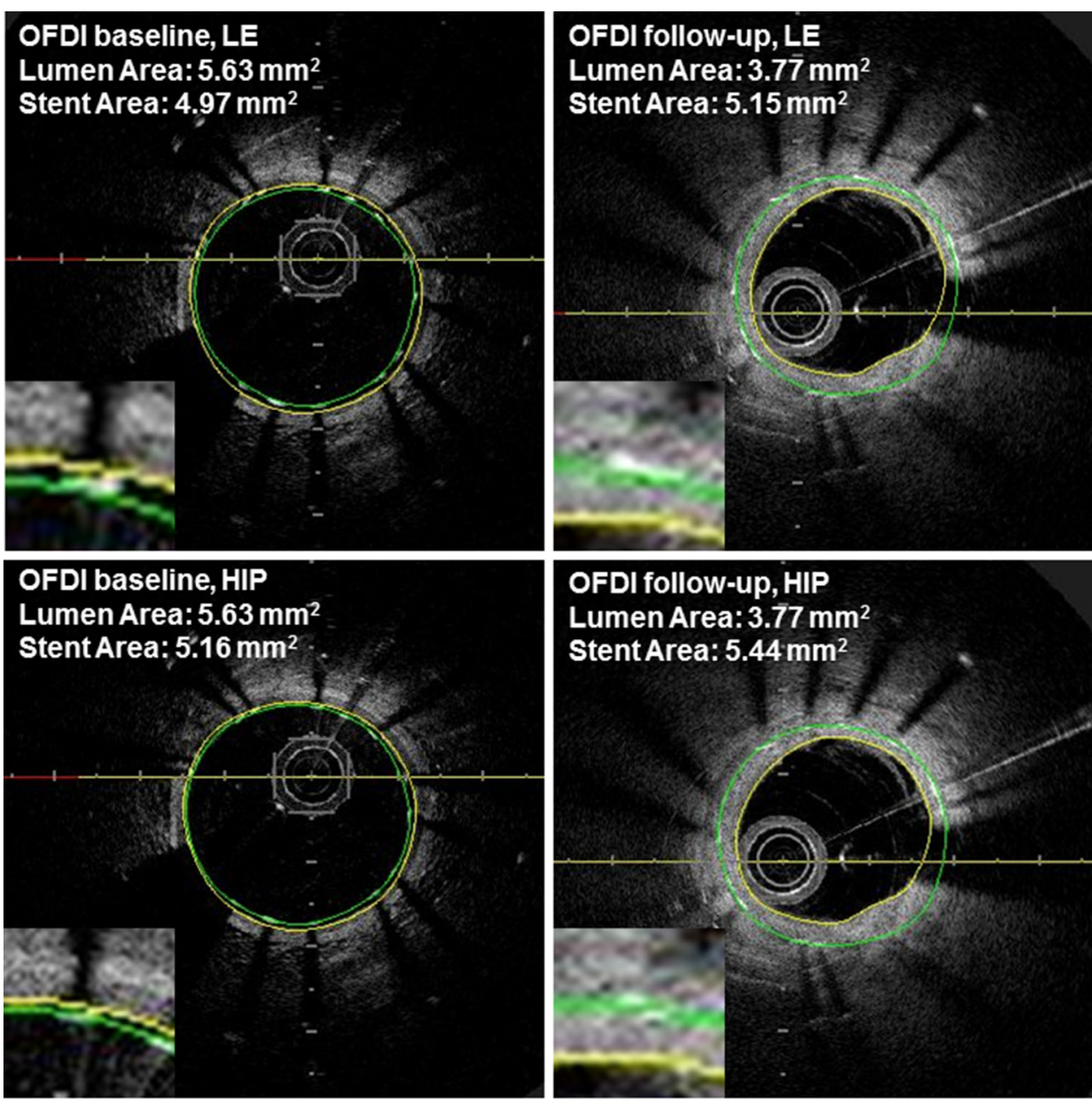

\section{OFDI follow-up, HIP Lumen Area: $3.77 \mathrm{~mm}^{2}$ Stent Area: $5.44 \mathrm{~mm}^{2}$}
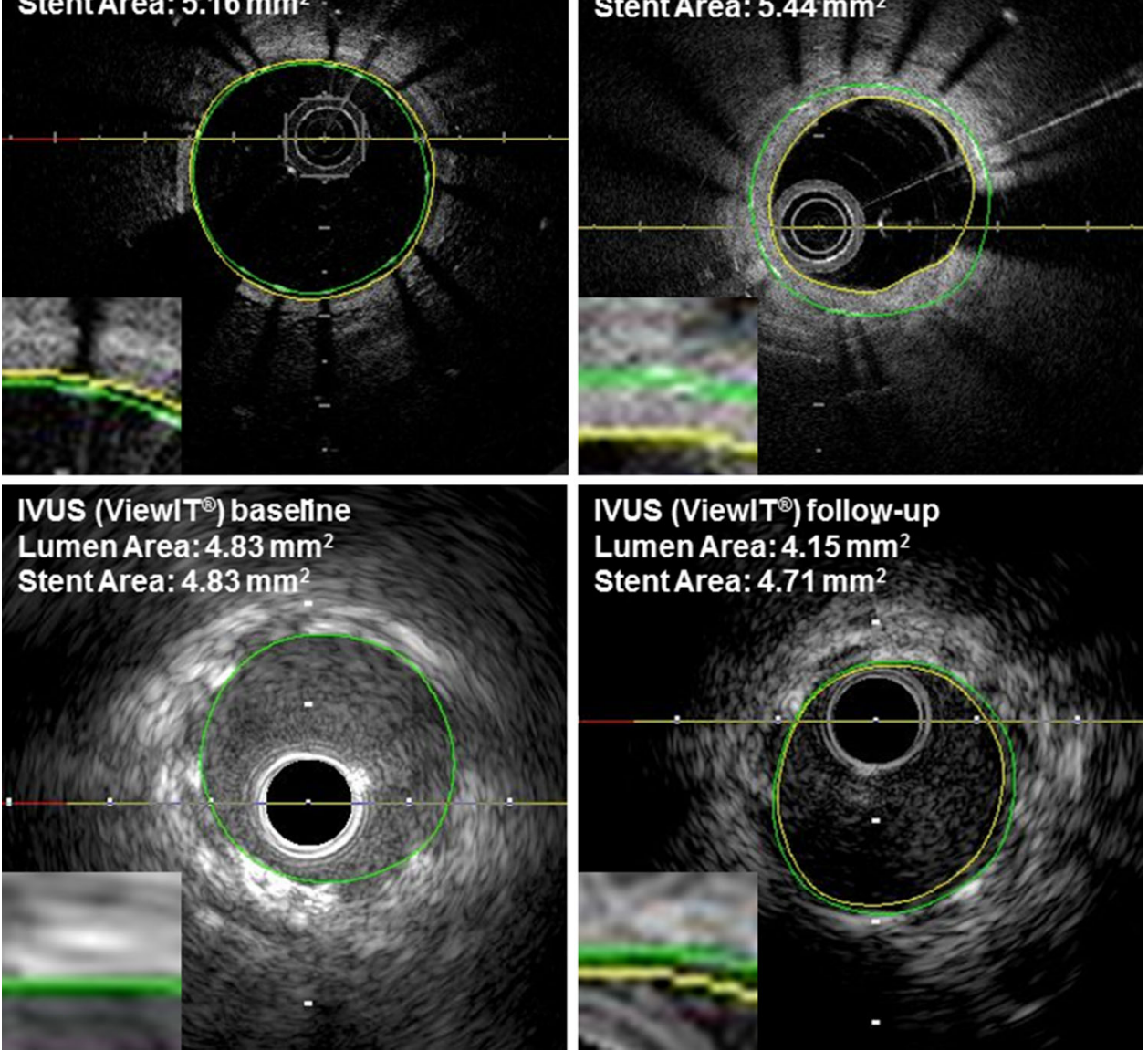

than IVUS at baseline, 4 weeks, and 12 weeks. As a result, overall stent measurements with this method were significantly larger in OFDI than in IVUS (difference in minimum stent area $=0.27 \pm 0.29 \mathrm{~mm}^{2}, P<0.001$; difference in stent VI $\left.=0.24 \pm 0.28 \mathrm{~mm}^{3} / \mathrm{mm}, P<0.001\right)$. For neointimal assessment at follow-up, OFDI consistently showed larger neointimal VI than IVUS both at 4 and 12 weeks. As expected, when the stent was traced at leading edges of strut images by OFDI, differences in calculated neointimal VI between OFDI and IVUS were numerically smaller compared to the tracing at highest intensity points (leading edges: $\quad$ difference $=0.16 \pm 0.21 \mathrm{~mm}^{3} / \mathrm{mm}, \quad P<0.001$; highest intensity points: difference $=0.45 \pm 0.19 \mathrm{~mm}^{3} /$ $\mathrm{mm}, P<0.001)$. 
Table 1 Comparisons of phantom mean lumen diameter (LD) by OFDI, FD-OCT, and IVUS (saline versus adjusted)

\begin{tabular}{llllll}
\hline & OFDI & FD-OCT & \multicolumn{2}{l}{ IVUS } & \\
\cline { 4 - 5 } & Saline & Saline & Saline & Adjusted & \% change \\
\hline $5.04 \mathrm{~mm}$ & $5.08 \pm 0.01$ & $5.04 \pm 0.01$ & $5.15 \pm 0.01$ & $5.04 \pm 0.01$ & -2.14 \\
$4.04 \mathrm{~mm}$ & $4.07 \pm 0.01$ & $4.05 \pm 0.01$ & $4.12 \pm 0.01$ & $4.04 \pm 0.01$ & -1.94 \\
$3.04 \mathrm{~mm}$ & $3.06 \pm 0.01$ & $3.07 \pm 0.01$ & $3.12 \pm 0.01$ & $3.06 \pm 0.01$ & -1.92 \\
$2.03 \mathrm{~mm}$ & $2.06 \pm 0.01$ & $2.04 \pm 0.01$ & $2.10 \pm 0.02$ & $2.07 \pm 0.02$ & -1.43 \\
$1.51 \mathrm{~mm}$ & $1.51 \pm 0.01$ & $1.49 \pm 0.01$ & $1.58 \pm 0.01$ & $1.56 \pm 0.01$ & -1.27 \\
\hline
\end{tabular}

$\%$ change was calculated as follows: $100 *$ (adjusted mean LD - mean LD in saline)/mean LD in saline $F D$-OCT frequency domain optical coherence tomography, IVUS intravascular ultrasound, $L D$ lumen diameter, $O F D I$ optical frequency domain imaging

Table 2 Comparisons of phantom mean lumen diameter (LD) by OFDI and FD-OCT in various conditions

\begin{tabular}{|c|c|c|c|c|c|c|c|c|}
\hline & \multicolumn{4}{|l|}{ OFDI } & \multicolumn{4}{|l|}{ FD-OCT } \\
\hline & Saline & $\begin{array}{l}\text { Saline } 50 \% \text {, } \\
\text { contrast } 50 \%\end{array}$ & Contrast & $\%$ change & Saline & $\begin{array}{l}\text { Saline } 50 \% \text {, } \\
\text { contrast } 50 \%\end{array}$ & Contrast & $\%$ change \\
\hline $5.04 \mathrm{~mm}$ & $5.08 \pm 0.01$ & $5.08 \pm 0.01$ & $5.05 \pm 0.01$ & 0.59 & $5.04 \pm 0.01$ & $5.03 \pm 0.01$ & $5.03 \pm 0.01$ & 0.20 \\
\hline $4.04 \mathrm{~mm}$ & $4.07 \pm 0.01$ & $4.07 \pm 0.01$ & $4.05 \pm 0.01$ & 0.49 & $4.05 \pm 0.01$ & $4.06 \pm 0.02$ & $4.04 \pm 0.01$ & 0.49 \\
\hline $3.04 \mathrm{~mm}$ & $3.06 \pm 0.01$ & $3.06 \pm 0.01$ & $3.07 \pm 0.01$ & 0.33 & $3.07 \pm 0.01$ & $3.08 \pm 0.01$ & $3.06 \pm 0.01$ & 0.65 \\
\hline $2.03 \mathrm{~mm}$ & $2.06 \pm 0.01$ & $2.05 \pm 0.01$ & $2.07 \pm 0.01$ & 0.97 & $2.04 \pm 0.01$ & $2.08 \pm 0.01$ & $2.04 \pm 0.01$ & 1.96 \\
\hline $1.51 \mathrm{~mm}$ & $1.51 \pm 0.01$ & $1.52 \pm 0.01$ & $1.53 \pm 0.00$ & 1.32 & $1.49 \pm 0.01$ & $1.53 \pm 0.01$ & $1.48 \pm 0.01$ & 3.36 \\
\hline
\end{tabular}

$\%$ change was calculated as follows: $100 *$ (maximum mean LD - minimum mean LD among three flushing media)/mean LD in saline $F D$-OCT frequency domain optical coherence tomography, $L D$ lumen diameter, $O F D I$ optical frequency domain imaging

\section{y-axis=mean LD by each modality-phantom diameter}
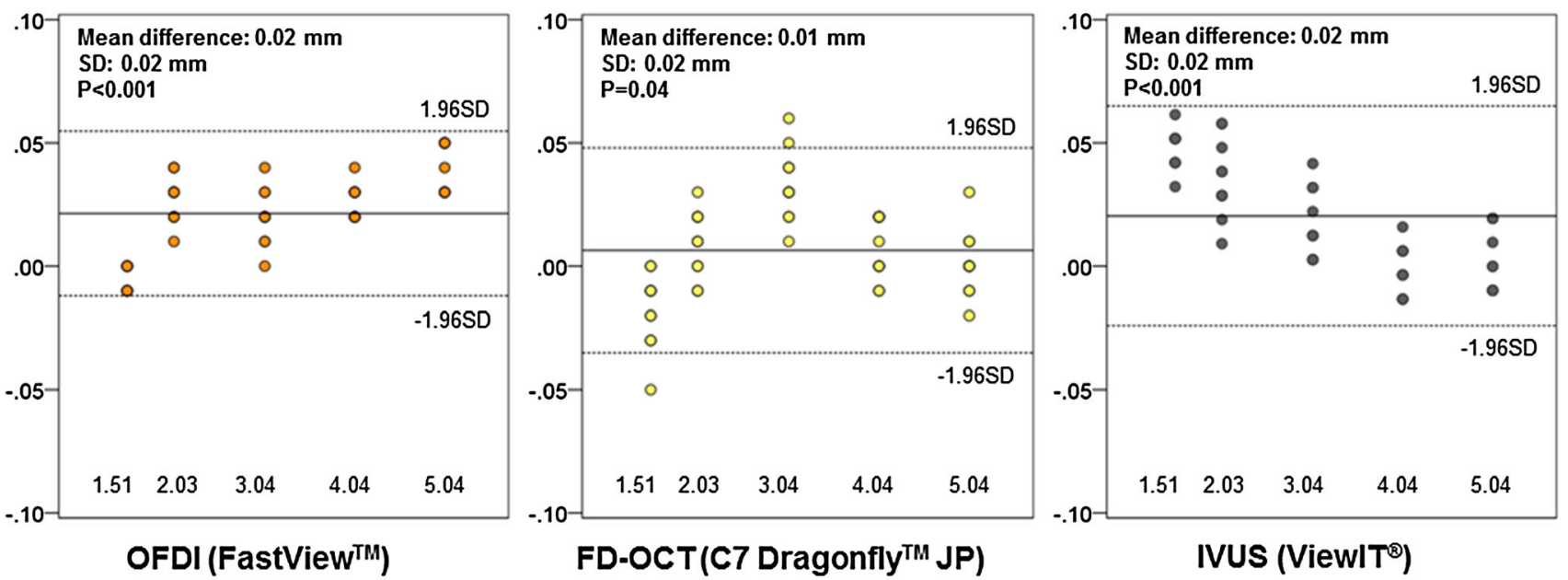

\section{x-axis=phantom diameters}

Fig. 3 Bland-Altman analysis for mean lumen diameter (in vitro study). In each panel, $x$-axis represents phantom diameter (1.51-5.04 mm) and y-axis represents the difference of measurement compared with phantom (mean LD by each modality-phantom diameter). Solid line shows mean value of LD difference in each modality, and two dotted lines show $\pm 1.96 \mathrm{SD}$ of mean LD difference in each modality. IVUS mean lumen diameters are adjusted values. $F D$-OCT frequency domain optical coherence tomography, IVUS intravascular ultrasound, $L D$ lumen diameter, $O F D I$ optical frequency domain imaging, $S D$ standard deviation 
Table 3 In vivo quantitative measurement by OFDI and IVUS at baseline, 4, and 12 weeks

\begin{tabular}{|c|c|c|c|c|c|c|c|c|c|}
\hline \multirow{2}{*}{$\begin{array}{l}\text { Total number of stents: } \\
\text { Coronary artery } \\
\text { (LAD/LCx/RCA): }\end{array}$} & \multicolumn{3}{|l|}{$\begin{array}{l}\text { Baseline } \\
34 \\
12 / 11 / 11\end{array}$} & \multicolumn{3}{|l|}{$\begin{array}{l}4 \text { weeks } \\
17 \\
7 / 3 / 7\end{array}$} & \multicolumn{3}{|l|}{$\begin{array}{l}12 \text { weeks } \\
13 \\
5 / 4 / 4\end{array}$} \\
\hline & OFDI & IVUS & $P$ value & OFDI & IVUS & $P$ value & OFDI & IVUS & $P$ value \\
\hline Mean LD (mm) & $2.65 \pm 0.26$ & $2.51 \pm 0.24$ & $<0.001$ & $2.31 \pm 0.42$ & $2.30 \pm 0.35$ & 0.96 & $1.86 \pm 0.34$ & $1.94 \pm 0.32$ & 0.002 \\
\hline $\begin{array}{l}\text { Minimum lumen area } \\
\left(\mathrm{mm}^{2}\right)\end{array}$ & $5.15 \pm 0.96$ & $4.73 \pm 0.95$ & $<0.001$ & $3.29 \pm 1.30$ & $3.86 \pm 1.15$ & 0.001 & $2.25 \pm 0.87$ & $2.48 \pm 0.82$ & 0.001 \\
\hline Lumen VI $\left(\mathrm{mm}^{3} / \mathrm{mm}\right)$ & $5.50 \pm 1.11$ & $4.95 \pm 0.99$ & $<0.001$ & $4.27 \pm 1.46$ & $4.22 \pm 1.26$ & 0.49 & $2.76 \pm 0.93$ & $3.00 \pm 0.96$ & 0.002 \\
\hline $\begin{array}{l}\text { Minimum stent area, } \\
\text { tracing at LE }\left(\mathrm{mm}^{2}\right)\end{array}$ & $4.63 \pm 0.89$ & $4.73 \pm 0.95$ & 0.02 & $5.05 \pm 1.21$ & $4.81 \pm 1.09$ & 0.002 & $4.69 \pm 0.83$ & $4.83 \pm 0.86$ & 0.03 \\
\hline $\begin{array}{l}\text { Minimum stent area, } \\
\text { tracing at HIP }\left(\mathrm{mm}^{2}\right)\end{array}$ & $4.92 \pm 0.94$ & $4.73 \pm 0.95$ & $<0.001$ & $5.34 \pm 1.27$ & $4.81 \pm 1.09$ & $<0.001$ & $4.98 \pm 0.87$ & $4.83 \pm 0.86$ & 0.03 \\
\hline $\begin{array}{l}\text { Stent VI, tracing at LE } \\
\left(\mathrm{mm}^{3} / \mathrm{mm}\right)\end{array}$ & $4.82 \pm 0.92$ & $4.95 \pm 0.99$ & $<0.001$ & $5.20 \pm 1.21$ & $4.95 \pm 1.15$ & $<0.001$ & $4.87 \pm 0.89$ & $4.98 \pm 0.92$ & 0.08 \\
\hline $\begin{array}{l}\text { Stent VI, tracing at HIP } \\
\left(\mathrm{mm}^{3} / \mathrm{mm}\right)\end{array}$ & $5.07 \pm 0.96$ & $4.95 \pm 0.99$ & 0.001 & $5.49 \pm 1.27$ & $4.95 \pm 1.15$ & $<0.001$ & $5.16 \pm 0.93$ & $4.98 \pm 0.92$ & 0.01 \\
\hline $\begin{array}{l}\text { Neointimal VI, tracing } \\
\text { at } \mathrm{LE}\left(\mathrm{mm}^{3} / \mathrm{mm}\right)\end{array}$ & N/A & N/A & N/A & $0.93 \pm 0.45$ & $0.73 \pm 0.32$ & 0.002 & $2.11 \pm 0.55$ & $1.98 \pm 0.63$ & 0.01 \\
\hline $\begin{array}{l}\text { Neointimal VI, tracing } \\
\text { at } \mathrm{HIP}\left(\mathrm{mm}^{3} / \mathrm{mm}\right)\end{array}$ & N/A & N/A & N/A & $1.22 \pm 0.43$ & $0.73 \pm 0.32$ & $<0.001$ & $2.40 \pm 0.58$ & $1.98 \pm 0.63$ & $<0.001$ \\
\hline
\end{tabular}

$H I P$ highest intensity points, IVUS intravascular ultrasound, $L A D$ left anterior descending artery, $L C x$ left circumflex artery, $L D$ lumen diameter, $L E$ leading edge, $O F D I$ optical frequency domain imaging, $R C A$ right coronary artery, $V I$ volume index

Intra- and interobserver reproducibility of OFDI was consistent, irrespective of parameters (coefficient of variation: $0.8-3.0 \%$ ). On the other hand, IVUS showed varying degrees of intra- and interobserver reproducibility among the parameters (coefficient of variation: 4.1-16.3\% for lumen, 0.6-3.4\% for stent, and 19.4-22.9\% for neointimal) (Table 4).

\section{Discussion}

The primary findings of this study are that OFDI, FD-OCT, and IVUS performed with clinically acceptable precision across a wide range of phantom diameters, and that in vivo measurements by OFDI and IVUS could slightly be discrepant depending on the parameters and time points after stent deployment (i.e., baseline and follow-up). The secondary finding is that the 2 methods for OFDI strut tracing (connecting leading edges vs. the highest intensity points) led to a small but systematic difference in measurement results.

Although several previous studies have attempted to compare OFDI or FD-OCT with conventional coronary imaging modalities (e.g., IVUS or quantitative coronary angiography), [2-4, 14, 15] no systematic validation existed with direct comparison between the 2 new intravascular optical imaging systems. To the best of our knowledge, the present study is the first to confirm comparability in quantitative assessment among OFDI, FD-OCT, and IVUS using latest-generation catheter systems tested in a variety of phantom diameters. Additionally, possible impact of different flushing media has also been evaluated in the present study. The refractive index for each flushing media has been confirmed to be appropriately defined by both OFDI and FD-OCT systems, suggesting that different flushing media can be selected in a given study according to coronary anatomy (e.g., arterial size, location, etc.) and clinical situations (e.g., renal insufficiency, etc.) as long as the reflective index of flushing media was adjusted by the system setting.

Despite the equivalent lumen measurements between OFDI and IVUS in the ideal experimental models, several discrepancies were observed in vivo between those 2 modalities, seemingly affected by multiple factors. First, lumen measurements at baseline were larger by OFDI than IVUS, most likely due to the difference of methodology in lumen tracing between the optical and ultrasound-based approaches (i.e., at baseline, lumen border behind stent struts can be visualized and traced by OFDI, whereas lumen and stent contours are virtually equal in IVUS in absence of incomplete strut apposition). On the other hand, as neointimal tissue proliferated within the stents during the follow-up, lumen measurements by OFDI became similar or smaller compared with IVUS, 

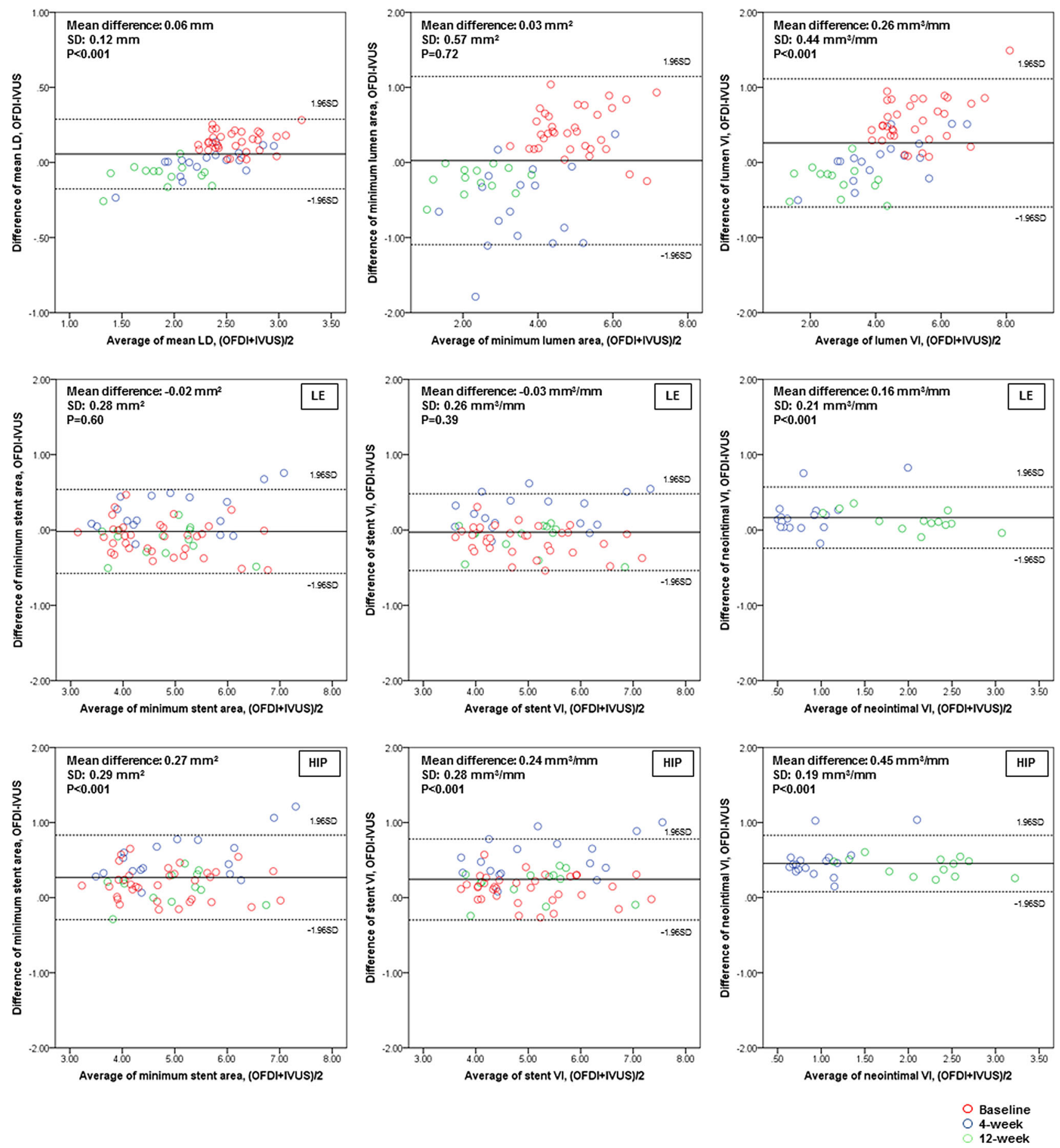

Fig. 4 Bland-Altman analysis for OFDI/IVUS parameters (in vivo study). In each panel, $x$-axis represents the average of measurement between OFDI and IVUS, and $y$-axis represents the difference of measurement between OFDI and IVUS (measurement by OFDImeasurement by IVUS). Solid line shows mean value of LD difference in each modality, and two dotted lines show $\pm 1.96 \mathrm{SD}$

which is in agreement with previous reports comparing FD-OCT [1, 3, 15] or OFDI [4] with IVUS. Another possible factor that can affect in vivo results may be the of difference between two modalities. IVUS intravascular ultrasound, $L D$ lumen diameter, $O F D I$ optical frequency domain imaging, $S D$ standard deviation, $V I$ volume index. $H I P$ and $L E$ indicate the stent trace in OFDI image was performed either at highest intensity points or at leading edges

effects of variance in blood flow velocity and temperature on IVUS measurements [16]. In addition, with imaging under flowing blood, IVUS may not always be able to 
Table 4 Intra- and interobserver variability in assessing swine coronary segments by OFDI and IVUS

\begin{tabular}{|c|c|c|c|c|}
\hline & \multicolumn{2}{|l|}{ Intraobserver variability } & \multicolumn{2}{|l|}{ Interobserver variability } \\
\hline & $\begin{array}{l}\text { Mean difference between } \\
1 \text { st and } 2 \text { nd time analysis }\end{array}$ & $\begin{array}{l}\text { Coefficient of } \\
\text { variation }(\%)\end{array}$ & $\begin{array}{l}\text { Mean difference between } \\
1 \text { st and 2nd observer }\end{array}$ & $\begin{array}{l}\text { Coefficient of } \\
\text { variation }(\%)\end{array}$ \\
\hline \multicolumn{5}{|l|}{ OFDI } \\
\hline Mean LD (mm) & $0.02 \pm 0.02$ & 1.1 & $0.04 \pm 0.02$ & 0.9 \\
\hline Minimum lumen area $\left(\mathrm{mm}^{2}\right)$ & $0.06 \pm 0.08$ & 2.6 & $0.14 \pm 0.06$ & 2.0 \\
\hline Lumen VI $\left(\mathrm{mm}^{3} / \mathrm{mm}\right)$ & $0.07 \pm 0.09$ & 2.4 & $0.12 \pm 0.09$ & 2.3 \\
\hline Minimum stent area, tracing at $\mathrm{LE}\left(\mathrm{mm}^{2}\right)$ & $-0.03 \pm 0.06$ & 1.4 & $-0.04 \pm 0.07$ & 1.6 \\
\hline Minimum stent area, tracing at $\operatorname{HIP}\left(\mathrm{mm}^{2}\right)$ & $-0.05 \pm 0.09$ & 1.9 & $0.01 \pm 0.07$ & 1.4 \\
\hline Stent VI, tracing at $\mathrm{LE}\left(\mathrm{mm}^{3} / \mathrm{mm}\right)$ & $-0.03 \pm 0.04$ & 0.9 & $-0.09 \pm 0.04$ & 0.8 \\
\hline Stent VI, tracing at HIP $\left(\mathrm{mm}^{3} / \mathrm{mm}\right)$ & $-0.04 \pm 0.04$ & 0.8 & $-0.03 \pm 0.05$ & 1.0 \\
\hline Neointimal VI, tracing at LE $\left(\mathrm{mm}^{3} / \mathrm{mm}\right)$ & $-0.05 \pm 0.06$ & 3.0 & $-0.16 \pm 0.05$ & 2.4 \\
\hline Neointimal VI, tracing at $\mathrm{HIP}\left(\mathrm{mm}^{3} / \mathrm{mm}\right)$ & $-0.07 \pm 0.07$ & 3.0 & $-0.09 \pm 0.07$ & 3.5 \\
\hline \multicolumn{5}{|l|}{ IVUS } \\
\hline Mean LD (mm) & $-0.02 \pm 0.09$ & 4.1 & $0.003 \pm 0.11$ & 5.4 \\
\hline Minimum lumen area $\left(\mathrm{mm}^{2}\right)$ & $-0.10 \pm 0.47$ & 14.1 & $0.02 \pm 0.53$ & 16.3 \\
\hline Lumen VI ( $\left.\mathrm{mm}^{3} / \mathrm{mm}\right)$ & $-0.08 \pm 0.26$ & 7.2 & $-0.002 \pm 0.34$ & 9.5 \\
\hline Minimum stent area $\left(\mathrm{mm}^{2}\right)$ & $0.01 \pm 0.10$ & 2.1 & $0.18 \pm 0.16$ & 3.4 \\
\hline Stent VI $\left(\mathrm{mm}^{3} / \mathrm{mm}\right)$ & $0.00 \pm 0.06$ & 1.2 & $0.001 \pm 0.03$ & 0.6 \\
\hline Neointimal VI $\left(\mathrm{mm}^{3} / \mathrm{mm}\right)$ & $0.12 \pm 0.34$ & 19.4 & $0.005 \pm 0.42$ & 22.9 \\
\hline
\end{tabular}

Second observation by same investigator was performed at least 4 weeks later after $1^{\text {st }}$ observation

$H I P$ highest intensity points, IVUS intravascular ultrasound, $L D$ lumen diameter, $L E$ leading edge, $O F D I$ optical frequency domain imaging, $V I$ volume index

detect the precise lumen border due to less contrast between blood speckles and intimal tissue, compared with optical imaging performed exclusively under blood removal. This hypothesis appeared to be supported by the higher intra- and interobserver variability of IVUS in assessing lumen and neointima than in assessing stent dimensions observed in the present study. Of note, however, this factor may have been exaggerated in the present study, performed in swine coronary arteries typically with different heart rates and shape of red blood cells compared with humans. Contrariwise, unsatisfactory blood removal during OFDI imaging could also lead to underestimation of the lumen size by making the differentiation of residual blood and lumen border challenging. Finally, differences in frame rate and pullback speed between the optical and ultrasound-based approaches may also have affected the in vivo results, leading to variances in image construction of the given coronary segment. From a clinical perspective, the differences observed in the present study may not necessarily lead to the alteration of interventional strategies, since the net difference in lumen measurements between OFDI and IVUS was small, irrespective of timing of observation.

To date, the methodology for stent analysis by OFDI/ FD-OCT has been under-investigated and controversial.
The Consensus Standards by the International Working Group recommend to trace at leading edges or the axial center of the stent strut images, though there has been no decisive evidence thus far [7]. The present study showed that tracing at leading edges had better agreement with IVUS-derived stent measurements than tracing at the highest intensity points; however, this result should not be misinterpreted as preference of one method over the other since no gold standard was available for the in vivo stent assessments. In fact, theoretically, the highest intensity points of strut images should correspond to the surface of metal struts more precisely than the leading edges that can include blooming artifacts often associated with strongly reflective metallic stents [7, 17]. Given that stent/neointimal values between the 2 methods are different to some extent, one would expect consistency in the methodology of tracing stent struts to be of importance to minimize possible systematic variability among the given cohort.

Another hypothesis-provoking observation from our in vivo stent analysis is that the difference in stent measurements between OFDI and IVUS appeared to be larger at 4 weeks than at baseline or 12 weeks, possibly suggesting that the amount and/or tissue characteristics of instent neointima may affect stent measurements. Since current intravascular optical imaging systems, including 
OFDI and FD-OCT, are calibrated to measure a distance from an imaging probe to a luminal border through a given flushing medium using the refractive index of the particular medium, the presence of neointima between the probe and stent struts may affect measurements by not taking the refractive index of neointima into account. Technically, IVUS measurements can also be affected by the different speed of sound in blood versus neointima, although ultrasound-based approaches may not be as sensitive as optical approaches to this phenomenon. The difference in the speed of sound between blood and soft tissue $(1540 \mathrm{~m} / \mathrm{s}$ on average) [18] is only $1.5 \%$, while the difference in saline and blood is $2.6 \%$ that resulted in merely $1-2 \%$ differences in IVUS-measured diameters in our phantom models. The exact refractive index of soft tissue for OFDI/FD-OCT is currently unknown, and therefore, this hypothesis warrants further investigation.

\section{Limitations}

Several limitations should be noted in this study. First, deviation of the imaging catheter from the center of the phantom may affect the measurements of in vitro study, although the catheter was positioned coaxially in the center of each phantom as much as possible and we employed the mean LD to minimize this limitation. Second, we employed the algorithm for IVUS data adjustment to avoid possible variability caused by varying blood characteristics in phantom models; however, this theoretical method is yet to be validated. Finally, the in vivo study was performed in swine coronary arteries without significant atherosclerosis but with significant in-stent neointimal proliferation, [19] the results of which may be somewhat different from those expected in diseased human coronary lesions successfully treated with drug-eluting stents.

\section{Conclusions}

OFDI, FD-OCT, and IVUS showed clinically acceptable precision across a wide range of phantom diameters. In vivo measurements by OFDI and IVUS could slightly be discrepant depending on the parameters and time points after stent deployment, although most differences appeared clinically irrelevant. In-stent assessment by OFDI, tracings at leading edges vs. the highest intensity points of strut images led to a small but systematic difference; therefore, consistency in methodology is advised for comparative studies.

Acknowledgments The authors thank Atsushi Hirohata, M.D., Ph.D. at Sakakibara Heart Institute of Okayama for his assistance with data acquisition, and Heidi N. Bonneau, RN, MS, CCA for her editorial review of the manuscript.

\section{Compliance with ethical standards}

Funding sources This study was partly supported by Terumo Corporation, Tokyo, Japan.

Disclosures All the authors have nothing to disclose regarding this manuscript.

Animal rights statement All procedures performed in studies involving animals were in accordance with the ethical standards of the institution or practice at which the studies were conducted.

\section{References}

1. Gonzalo N, Serruys PW, Garcia-Garcia HM, van Soest G, Okamura T, Ligthart J, et al. Quantitative ex vivo and in vivo comparison of lumen dimensions measured by optical coherence tomography and intravascular ultrasound in human coronary arteries. Rev Esp Cardiol. 2009;62:615-24.

2. Tahara S, Bezerra HG, Baibars M, Kyono H, Wang W, Pokras S, et al. In vitro validation of new Fourier-domain optical coherence tomography. EuroIntervention. 2011;6:875-82.

3. Kubo T, Akasaka T, Shite J, Suzuki T, Uemura S, Yu B, et al. OCT compared with IVUS in a coronary lesion assessment: the OPUS-CLASS study. JACC Cardiovasc Imaging. 2013;6:1095104.

4. Okamura T, Onuma Y, Garcia-Garcia HM, van Geuns RJ, Wykrzykowska JJ, Schultz C, et al. First-in-man evaluation of intravascular optical frequency domain imaging (OFDI) of Terumo: a comparison with intravascular ultrasound and quantitative coronary angiography. EuroIntervention. 2011;6:1037-45.

5. Hiro T, Leung CY, Russo RJ, Karimi H, Farvid AR, Tobis JM. Variability of a three-layered appearance in intravascular ultrasound coronary images: a comparison of morphometric measurements with four intravascular ultrasound systems. Am J Card Imaging. 1996;10:219-27.

6. Yamada R, Okura H, Kume T, Hayashida A, Neishi Y, Kawamoto $\mathrm{T}$, et al. Comparison of quantitative measurements between two different intravascular ultrasound systems: in vitro and in vivo studies. J Cardiol. 2013;61:201-5.

7. Tearney GJ, Regar E, Akasaka T, Adriaenssens T, Barlis P, Bezerra HG, et al. Consensus standards for acquisition, measurement, and reporting of intravascular optical coherence tomography studies: a report from the International Working Group for Intravascular Optical Coherence Tomography Standardization and Validation. J Am Coll Cardiol. 2012;59:1058-72.

8. Greenspan M, Tschiegg CE (1957) J Natl Bureau of Standards p. 249.

9. Murata A, Wallace-Bradley D, Tellez A, Alviar C, Aboodi M, Sheehy A, et al. Accuracy of optical coherence tomography in the evaluation of neointimal coverage after stent implantation. JACC Cardiovasc Imaging. 2010;3:76-84.

10. Mintz GS, Nissen SE, Anderson WD, Bailey SR, Erbel R, Fitzgerald PJ, et al. American College of Cardiology Clinical Expert Consensus Document on Standards for Acquisition, Measurement and Reporting of Intravascular Ultrasound Studies (IVUS). A report of the American College of Cardiology Task Force on Clinical Expert Consensus Documents. J Am Coll Cardiol. 2001;37:1478-92. 
11. Bland JM, Altman DG. Statistical methods for assessing agreement between two methods of clinical measurement. Lancet. 1986;1:307-10.

12. van der Lugt A, Gussenhoven EJ, Pasterkamp G, Bom N, Posthuma DJ, Stijnen T. Interobserver reproducibility of qualitative and quantitative analysis of intravascular ultrasound images before and after peripheral balloon angioplasty. Ultrasound Med Biol. 1996;22:399-404.

13. Hibi K, Takagi A, Zhang X, Teo TJ, Bonneau HN, Yock PG, et al. Feasibility of a novel blood noise reduction algorithm to enhance reproducibility of ultra-high-frequency intravascular ultrasound images. Circulation. 2000;102:1657-63.

14. Kim SJ, Lee H, Kato K, Yonetsu T, Jang IK. In vivo comparison of lumen dimensions measured by time domain-, and frequency domain-optical coherence tomography, and intravascular ultrasound. Int J Cardiovasc Imaging. 2013;29:967-75.

15. Gonzalo N, Escaned J, Alfonso F, Nolte C, Rodriguez V, Jimenez-Quevedo $\mathrm{P}$, et al. Morphometric assessment of coronary stenosis relevance with optical coherence tomography: a comparison with fractional flow reserve and intravascular ultrasound. J Am Coll Cardiol. 2012;59:1080-9.

16. Chae JS, Brisken AF, Maurer G, Siegel RJ. Geometric accuracy of intravascular ultrasound imaging. J Am Soc Echocardiogr. 1992;5:577-87.

17. Guagliumi G, Costa MA, Sirbu V, Musumeci G, Bezerra HG, Suzuki N, et al. Strut coverage and late malapposition with paclitaxel-eluting stents compared with bare metal stents in acute myocardial infarction: optical coherence tomography substudy of the Harmonizing Outcomes with Revascularization and Stents in Acute Myocardial Infarction (HORIZONS-AMI) Trial. Circulation. 2011;123:274-81.

18. Omari E, Lee H, Varghese T. Theoretical and phantom based investigation of the impact of sound speed and backscatter variations on attenuation slope estimation. Ultrasonics. 2011;51: 758-67.

19. Virmani R, Kolodgie FD, Farb A, Lafont A. Drug eluting stents: are human and animal studies comparable? Heart. 2003;89: $133-8$. 